\title{
Traditional ecological knowledge and biodiversity conservation: the medicinal plants of the Dayak Krayan people in Kayan Mentarang National Park, Indonesia
}

\author{
RINA SUSANTI ${ }^{1, \vartheta}$, ERVIZAL A. M. ZUHUD ${ }^{2, \bullet \bullet}$ \\ ${ }^{1}$ Graduate School of Media and Governance, Keio University. Shonan Fujisawa Campus, 5322 Endo, Fujisawa-shi, Kanagawa-ken 252-0882, Japan \\ Tel./fax.: +81-466-49-3404, vemail: rsusanti@ sfc.keio.ac.jp \\ ${ }^{2}$ Faculty of Forestry and Environment, Institut Pertanian Bogor. Jl. Ulin, Kampus IPB Darmaga, Bogor 16680, West Java, Indonesia, \\ Tel/fax.: +62-812-1062154, ^vemail: ervizal_amzu@yahoo.com
}

Manuscript received: 19 June 2019. Revision accepted: 31 August 2019.

\begin{abstract}
Susanti R, Zuhud EAM. 2019. Traditional ecological knowledge and biodiversity conservation: the medicinal plants of the Dayak Krayan people in Kayan Mentarang National Park, Indonesia. Biodiversitas 20: 2764-2779. Traditional ecological knowledge and biodiversity conservation can support one another for management of national parks in Indonesia as some such areas established in customary areas. This quantitative ethnobotany study aims to find the correlation of traditional ecological knowledge of medicinal plants of the Dayak Krayan people to biodiversity conservation in Kayan Mentarang National Park (KMNP), North Kalimantan, Indonesia. The research method used key informant interviews, transect walks, and questionnaire interviews. There were about 51 and 42 medicinal plants in Wa' Yagung and $\mathrm{Pa}$ ' Padi respectively, which most used in life form of herb and tree, and to treat digestive system and infestations. Moreover, the important species are trees Alstonia scholaris and Cinnamomum cuspidatum, and vine Aristolochia sp. which grow in primary forest, while shrub Melastoma malabathricum in young secondary forest. There were significant differences in knowledge and use regarding age and gender groups, which older groups tend to possess higher values. The linking concept is proposed by using a review of three stimuli NUR (Natural-Usefulness-Religious) pro-conservation tool of traditional ecological knowledge and biodiversity conservation. The authorities' stakeholders in KMNP will have to emphasize the traditional ecological knowledge in short or long-term management plan to achieve the objective of collaborative management in this national park.
\end{abstract}

Keywords: Biodiversity conservation, Dayak, Kayan Mentarang National Park, Krayan, medicinal plants, traditional ecological knowledge

Abbreviations: WY: Wa' Yagung, PP: Pa' Padi, KMNP: Kayan Mentarang National Park, TEK: Traditional Ecological Knowledge

\section{INTRODUCTION}

Traditional ecological knowledge or TEK is knowledge or belief of people living in nature which is passed culturally to younger generations (Johnson 1992; Berkes 1993). Traditional ecological knowledge has been important in the management and conservation of forest areas (Posey 1997; Gerique 2006; SCBD 2006). Interdependency between local communities, biodiversity and their knowledge in managing medicinal plant resources has gained recognition in the world today (Ghimire and Bastakoti 2009). However, there is a threat as deforestation occurred in tropical regions calculated by FAO affected to loss of biodiversity, cultural assets and knowledge, which the knowledge mostly in oral tradition and subjected to biopiracy and violation of intellectual property rights (Balick and Cox 1997; Mayers and Bass 1998; Kathe 2006). While such loss of knowledge and biodiversity looks as irreversible, people may deter it (Kala 2000). There is also an often-stated assumption that the discovery of a new plant drug will undoubtedly help in conservation efforts, particularly in rain forest regions (Balick et al 1996). Also added by Sedjo (2000) that plant biodiversity may valuable as sources of natural products for medicine. In Indonesia, traditional ecological knowledge of medicinal plants is recognized by park and buffer zone communities as well as in remote rural areas where modern health care services are less (MoF 2002a). Medicinal plants knowledge is highly praised by Dayak people in the Krayan area as indicated by the presence of a respected local healer in Wa' Yagung village. The knowledge was developed by living in the forests for hundreds of years, therefore the Dayaks possess rich traditional knowledge.

Kayan Mentarang National Park (KMNP) has developed its area of 1.35 million hectares with diverse forest ecosystems collaboratively with the local people and the local government to accommodate their concerns. McNeely and MacKinnon (1990) agreed that local people and protected area managers can become allies in managing a natural reserve area. While managers can learn much about resource conservation and use, on the other hand, nature conservation supports the survival of traditional cultures. As to explain the biodiversity and the research in KMNP, at the southern part of KMNP, there is an area with higher elevation called Apo Kayan which research on traditional medicinal plants for malaria 
ailments was conducted by Leaman et al. (1995). A joint biodiversity expedition involving researchers from Indonesia and Malaysia was conducted in $\mathrm{Pa}$ 'Raye village of Krayan Hilir customary area at northern part, to study about park's biodiversity including ethnobotany (Matius et al. 2003). In KMNP, the Dayaks live in an area with diverse ecological characteristics. The southern region is tropical lowland moist forest which is inhabited by Dayak Kenyah people, while the northern part is montane and heath forests inhabited by Dayak Lundayeh people (Wulffrat 2005). Collaborative management has been aimed to manage the park with the principles of sharing responsibility, benefit, and role, as regulated in Ministry of Forestry of Indonesia decree number 1213, 1214, and 1215 of 2002 (MoF 2007), with some proposed options for participatory remapping and zonation of the park. This study aims to document the traditional ecological knowledge of medicinal plants of the Dayak traditional communities and to analyze its role in biodiversity conservation in collaboratively managed KMNP.

\section{MATERIALS AND METHODS}

\section{Research sites}

This study was conducted in two villages of Wa' Yagung and Pa'Padi, Krayan Sub-district, Nunukan District, North Kalimantan, Indonesia from May to June 2009 (part of East Kalimantan Province before 2013). The sites are located in $03^{\circ} 57^{\prime} 52^{\prime \prime} \mathrm{N}$ and $115^{\circ} 51^{\prime} 50^{\prime \prime} \mathrm{E}$, in the northern part of KMNP (Figure 1). Wa' Yagung village is culturally in Krayan Hilir customary area and located inside the Park, while $\mathrm{Pa}^{\prime}$ Padi is situated at the park boundary and is in Krayan Darat customary area. Krayan area is a highland plateau of 700-1,100 masl in elevation, the slope of $41-60 \%$, sedimentary mountain ridges, and forest types of secondary and heath forests with a dense structure of trees and shrubs (Langub 2005; Wulffrat 2005). Wa' Yagung village area is approximately $90 \%$ (59,647 ha) part of KMNP (MoF 2002b). Wa' Yagung River flows to Kemalun River of Krayan headwaters, the most important river in this area (Map). Wa' Yagung is inhabited by Lundayeh sub-group, the most dominant Dayak group of Krayan. It is the only village never experienced resettlement in Krayan, while other villages had such during the development at the beginning of the 1970 s as stated by Kapung 2009 (pers.comm.). This resettlement caused people to leave their remote small villages in the forest areas to regroup in relatively accessible areas, while still keep their own administrative systems. Wa' Yagung is the furthest village to access in Krayan. People will have to on foot for a distance of $18 \mathrm{~km}$ to Long Umung (the closest village as a hub) and followed by an hour of the motorcycle to Long Bawan (the capital of Krayan sub-district), while other villages are accessible by motorcycle. The village demographic databook (Buku Data Induk Kependudukan) in April 2009 served the data of 40 households and a total of 156 persons, with 91 males and 65 females (KSDG 2009). Pa'Padi area covers 7,836 ha, and about 357 ha or $5 \%$ is part of the KMNP area $(\mathrm{MoF}$ 2002b). Unlike Wa' Yagung, Pa Padi is resettlement of two villages of $\mathrm{Pa}$ Padi and Cinglat since the 1970s, and people in Pa' Padi speak their own language which different to the most spoken Lundayeh language (Matius et al. 2003 pers. comm.). It is relatively accessible from sub-district capital using motorcycles for an hour. The population in Pa'Padi in March 2009 was 65 households, with 99 females and 109 males (KSDG 2009).

Main livelihood in both villages based on the traditional practice of paddy field of Adan Krayan rice, hunting and gathering, handicraft from rattan, or work in Malaysia. Information collected during the focus group interview of Group older males in Pa' Padi on June 10th, 2009, the Dayak sub-ethnic divided into two groups associated with their agricultural practices. The first is called Tana' Lun for those live at higher elevation areas, therefore, practice swidden agriculture. On the other hand, the second is Lun $\mathrm{Ba}^{\prime}$, people live at lower elevation areas, therefore, viable for wet-rice agriculture. While Wa' Yagung classified as Lun $\mathrm{Ba}^{\prime}$ and speak common Lundayeh language called bahasa lurus (straight language), Pa'Padi called themselves as the third group as they speak a particular language. Regardless of people in Krayan speak their language daily, they understand well of Indonesian. People had to head to Long Bawan for staple goods and fuel imported from Ba'Kelalan, Malaysia, or to sell handicrafts. There were pioneer flights to and from Long Bawan with the subsidy, however, it was limited with the priority for the villagers who need to make reservation a month before or healthcare-needed villagers. However, villagers had to book an extra flight with no subsidy. Sometimes a flight canceled due to weather or technical reason. Each village has one elementary school, therefore for higher education, the students had to continue their education to junior high school at Long Umung or Long Bawan, or high school or a theology college in Long Bawan, or other cities. One public health facility is at the sub-district capital, which one doctor and nurses visit the villages every month. There was a visit for women's anti-tetanus vaccination when the research conducted in $\mathrm{Wa}^{\prime}$ Yagung.

Coping these constraints, the Dayaks are forest communities which have been practicing traditional knowledge for daily necessities using forest resource, in particular for health care using plant species from the forest. However, there is a concern as this knowledge has been passed on through generation by verbal culture.

\section{Research methods}

In this quantitative ethnobotany method, the data were obtained in Wa' Yagung by interviewing a prominent local healer, Daniel Kapung, while in Pa' Padi employed a focus group discussion with a group of men. The informants in Pa' Padi were the knowledgable villagers recommended and invited by the head of the village, Martin, as this village had no particular local healer. These data of vernacular names used as the catalog questionnaire to interview the respondents (villagers) to assess the significance of use and knowledge among the people in groups based on gender and age. 


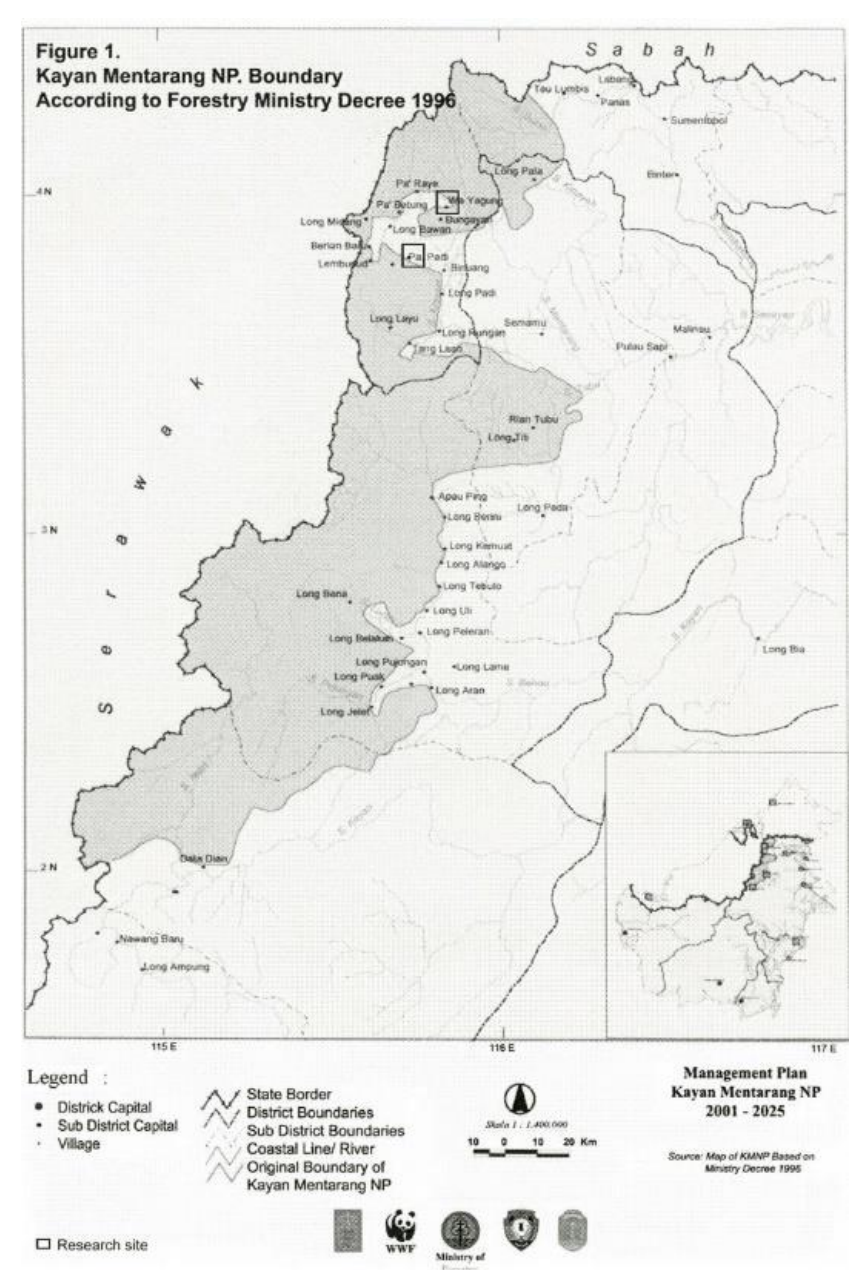

Figure 1. Location of study (MoF 2002)

Data on uses and preparation recorded by several interviews with a local healer and some villagers both men and women. Direct observation was done in participating in communal activities. This ethnobotanical method is for determining the important species which possibly for future development using some guidelines from Martin (1998) on ethnobotany data collection, and rapid and participatory rural appraisal (Furze et al. 1996; Townsley 1996). The different catalogs of medicinal plants species from both villages intended to discover and to document the medicinal plants of Wa' Yagung and $\mathrm{Pa}^{\prime}$ Padi as both villages are different in customary land area, language, and site ecology. Transect walks were followed after the approval of vernacular names of medicinal plants species mentioned during the interviews and discussions, to collect plant specimens guided by the villagers. Collecting voucher specimens is important as primary sources for proof of plants' resources at geographic range and habitat types (Foote and Jones 1989). Transect walks accomplished in several days to collect plant specimens and to record the data of characteristic of the medicinal plant species in their habitats, the habitat description, coordinate, elevation, and images in the field. Data and images of the plants in their habitat gathered during transect walks were important in interviewing the villagers and for identification at
Herbarium Samboja of Ministry of Forestry. The plant specimens were collected as wet specimens for two replicates in mostly fertile condition and minimum disturbance. Some common herb species were collected without voucher specimens considering the limited baggage using pioneer flight.

\section{Data analysis}

Data of medicinal plants species used by villagers in the last three years were collected from key informant interview, semi-structured and focus group interview, transect walk, direct observation, and secondary data review. Data analysis using tests of Kruskal-Wallis for among independent samples and Mann-Whitney between two independent samples (Morgan et al. 2007). Knowledge and use values are calculated to determine the local important species (LI), using people's knowledge and use of a species as medicine in the last three years. The calculation is $\mathrm{LI}=\mathrm{VU}+\mathrm{VK}$ where $\mathrm{VU}$ is the mean value of the use of a species by the villagers, and VK is the value of knowledge of a species by the villagers in four groups (Table 1). Three stimuli NUR (Natural-Use-Religious) proconservation by Zuhud (2007) and Zuhud et al. (2007) is used to review in accordance of natural stimulus (the natural bio-ecology character of the species), usefulness stimulus (the importance to the people: economic, medicine, and bio-ecological values), and religious stimulus (reviews the value related to god, religion, belief, and spiritual) based on observation during fieldwork.

\section{RESULTS AND DISCUSSIONS}

\section{Local important medicinal plants species}

According to the species identification by the experts at Herbarium Samboja, there were 51 species of 42 families in Wa' Yagung and 42 species of 33 families in Pa' Padi, specimens of Limuan, Rumput WY and Uduh Tageng Bara in Wa' Yagung, and Rumput Sepula'Air, and War Seruyung (Melastomataceae) unidentified (Table 1). Among them were 16 same species of Bua' Libuh and Va' Ibuh (Psidium guajava), Dinudur (Basella alba), Ideb (Cyathea borneensis), Kaki Kuda and Uduh Pelali (Centella asiatica), Kayu Lingat (Lansium domesticum), Keriboro Apad and Teboro Apad (Boesenbergia stenophylla), Kumis Kucing (Orthosiphon sp.), Pikutuh (Leucosyke capitellata), Sikeli' and Si'yang (Melastoma malabathricum), Tabar (Cinnamomum cuspidatum), Temawar and Petawar (Aristolochia sp.), Uduh Ame' (Ageratum houstonianum), Uduh Bulu' (Lophatherum gracile), Uduh Lepen and Kayu Pentenab (Maesa ramentacea), Wat Ulem (Solanum torvum), and We Keraruh (Calamus sp.) (Tabel 1). In Wa Yagung, species with $\mathrm{VK}=1.00$ (all the respondents are familiar with) were Babas/Brotowali (Tinospora crispa), Daun Sendok (Plantago major), Kumis Kucing (Orthosiphon sp.), and Sikeli' (Melastoma malabathricum). Other species with VK $>0.70$ were Angeh (Alstonia scholaris), Baku'Cat (Etlingera elatior), Daun Buyu' (Piper sp.), Lamak (Kalanchoe pinnata), Dinudur (Basella alba), Karab 
(Pronephrium rubicundum), Lab Menipal (Erycibe sp.), Merebung (Elmerillia tsiampacca), BuáLibuh (Psidium guajava), Pepaya (Carica papaya), Tabar (Cinnamomum cuspidatum), Temawar (Aristolochia sp.), Tutud Ada' (Tinospora sp.), Uduh Babeh Anak (Phyllanthus urinaria), and War Birar (Fibraurea tinctoria) (Tabel 1). In Pa'Padi, villagers acknowledged Si'yang (Melastoma malabathricum) and Tabar (Cinnamomum cuspidatum) as well, highly acknowledged species with $\mathrm{VK}>0.70$ are Fedeyur (Goniothalamus macrophylla), Kayu Langsat (Lansium domesticum), Kayu Lengiril (Macaranga costulata), Kayu Yureng (Fagraea racemosa), Kumis Kucing (Orthosiphon sp.), Pasak Bumi (Eurycoma longifolia), Petawar (Aristolochia sp.), Uduh Bulu' (Lophatherum gracile), Uduh Kerbau (Paspalum sp.), Uduh Pan (Begonia sp.), Uduh Pelali (Centella asiatica), Va'Tbuh (Psidium guajava), War Babas (Coscinium penestratum), War Seruyung (Melastomataceae), Wat Ulem (Solanum torvum), We Keraruh (Calamus sp.), and Ya'un Koko (Theobroma cacao). While some species with VU>0.70 were Angeh and Tabar in Wa' Yagung, and Petawar, Si'yang, also Tabar in Pa'Padi. Therefore, the local important (LI) medicinal plants species revealed three important medicinal plants species in $\mathrm{Wa}$ ' Yagung were Angeh (Alstonia scholaris) LI 1.70, Tabar (Cinnamomum cuspidatum) LI 1.68, and Temawar (Aristolochia sp.) LI 1.65. While in Pa'Padi, there were two species of Petawar (Aristolochia sp.) LI 1.83, and Tabar (Cinnamomum cuspidatum) LI 1.73, and Si'yang (Melastoma malabathricum) LI 1.77 (Table 1). Tree of Tabar (Cinnamomum cuspidatum) and the vine of Temawar (Aristolochia sp.) were all found in the primary forest, while the tree of Angeh (Alstonia scholaris) found in primary and young secondary forest observed in $\mathrm{Wa}$ ' Yagung. Shrubs of Si'yang (Melastoma malabathricum) found abundant in young secondary forest accessible around the village of Pa'Padi. Angeh (Alstonia scholaris) was not acknowledged in Pa'Padi, as no villagers informed and heard about this species. Practices in both villages give to assure the sustainability of such biodiversity as at current situation as both villages are in the lower population, customary law acknowledgment, no commercial purpose, and substitution properties by some species. Nevertheless, the sustainable harvesting methods must take account in the future when villages responding to the development in the area and population that increase the intensity of extraction presumably. The more concern way is debarking, as it causes a low opportunity for the individual to survive. Debarking is considered as a disturbance as stated by Bleher et al. (2006) who use it as one of the disturbance parameters in a survey in Kakamega forest, Kenya.

\section{Habitat and plants species}

Traditional land use/forest classification in both villages appeared to be as a common system in the Dayak Krayan communities as the similar was found in $\mathrm{Pa}$ 'Raye village of Krayan Hilir customary area (Matius et al. 2003). The Ransa Dayak ethnic in West Kalimantan also uses similar classification, only in different local names based on Caniago and Siebert (1998). In Wa' Yagung, most medicinal plants found in Amug Dari (young secondary forest) for $50.79 \%$, followed by Lati' (forest garden), Amug Kara (old secondary forest), Pulung Kara (primary forest) and $\mathrm{Ba}^{\prime}$ (rice field) (Table 3). Similar to Pa'Padi, Amug Dari (young secondary forest) was the highest with 18 species $(30.51 \%)$ followed by Amug Kara (old secondary forest), Pulung Kara (primary forest), Lati' (forest garden), and $\mathrm{Ba}^{\prime}$ (rice field) respectively (Table 3). This shows villagers utilize mostly species occur in Amug Dari (young secondary forest) which relatively at short walking distance. This local land-use type is generally at the vicinity of the village and has characteristics of the young secondary forest as it is fallow for less than 10 years. Therefore, it comprises of pioneer species and understorey. In agriculture management system, Dayak people of Pujungan customary area also practice 25-year rotation sustainable agriculture but in the form of swidden according to Iiyama (2003), while in Krayan there are no swidden practices.

There were 51 plants of 41 families, 47 genera and 35 species-level in Wa' Yagung, and 42 of 34 families, 39 genera, and 30 species-level in Pa Padi. Villagers used plants mostly to treat the disease in the digestive system and infestations. The highest number of medicinal plants species found in young secondary forest (Amug Dari) in both villages, which dominated by shade-intolerant early pioneers and the herbs. It is the characteristic of this part in Borneo of Kerayan-Kelabit which dense in the undergrowth and tends to lower number occurrence at higher altitudes of 1000-1280 meters (Schneeberger 1945). High occurrence of medicinal plants species in Amug Dari (young secondary forest) demonstrated the favorable distance accessing the medicinal plant's species for the villagers, for an emergency in particular, besides the abundance of daily useful species with the medicinal properties in this forest type. Wild plants species occur in higher number in forest garden (Lati') and rice field ( $\mathrm{Ba}^{\prime}$ ) showed Wa' Yagung which located deep in the forest and accessible only on foot, also low population causes the low intensity of anthropogenic disturbance. It explains the link between the diversity of plants and disturbance factors for medicinal plants derived by Schippmann et al. (2006) and added by Montagnini and Jordan (2005) where secondary succession often occurs in large areas of fallowed agricultural land without further disturbance. The term of a forest garden is to define the area around the village sowed with crops for diet needs, while $\mathrm{Ba}^{\prime}$ (wet rice paddy field) is the wet area of rice paddy for staple in an annual rotation permanently on an area. Upper montane forest situation in KMNP reflected in Pa'Padi which located at elevation $>950 \mathrm{~m}$ asl, and has characteristics of species, features of structure and appearance of heath forests, where the soils change according to altitude and generally more acidic and poor in nutrient (WWF 2007). Heath forest causes a low percentage in productivity as it has lower fertile soils (MacKinnon et al. 1996). This is likely the reason of low diversity of wild species at settlement area in Pa' Padi, besides higher number of population and anthropogenic intensity in $\mathrm{Pa}^{\prime}$ Padi. 
Table 1. Medicinal plants in Wa' Yagung and Pa' Padi, Kayan Mentarang National Park (KMNP), North Kalimantan, Indonesia

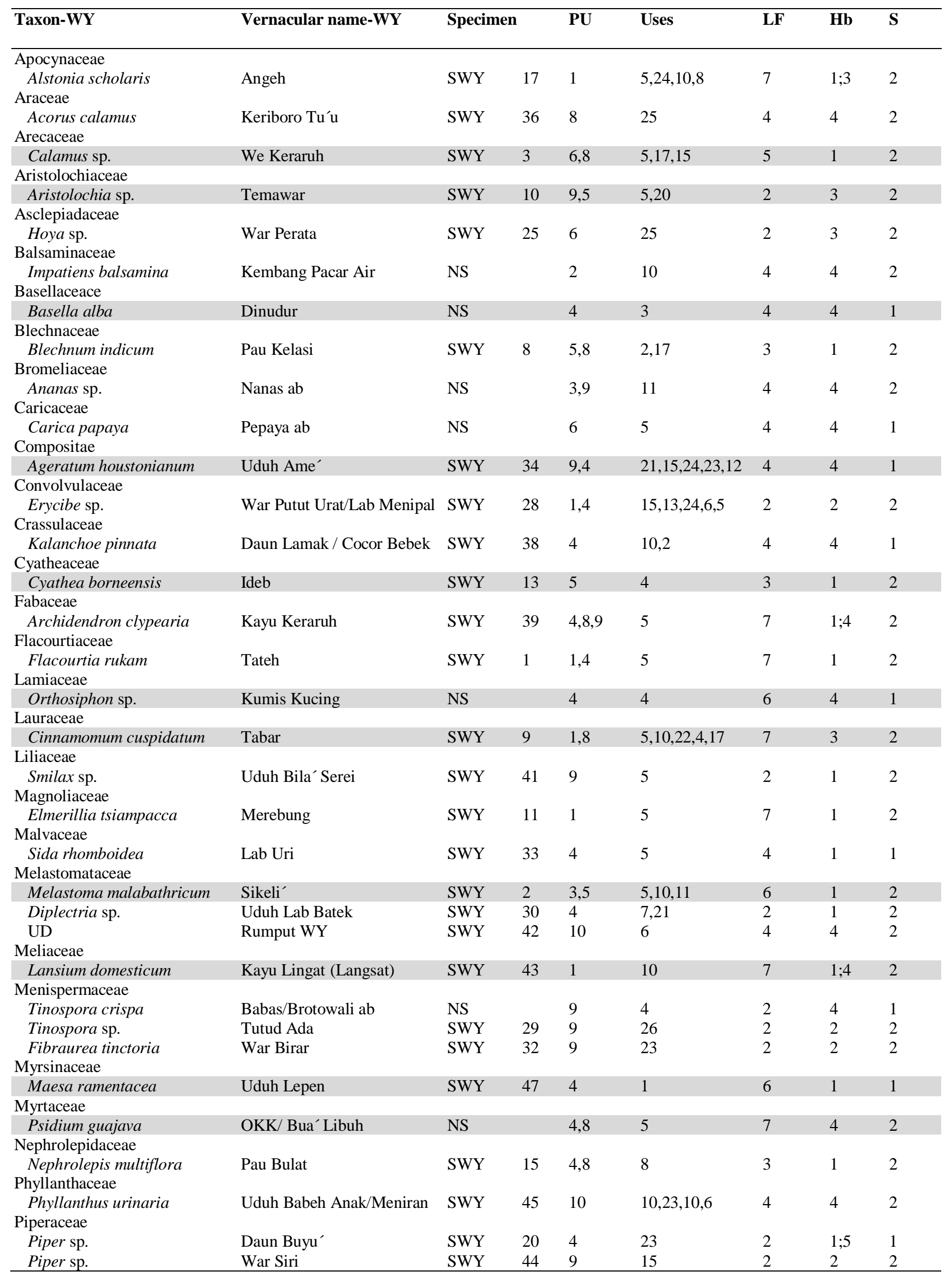




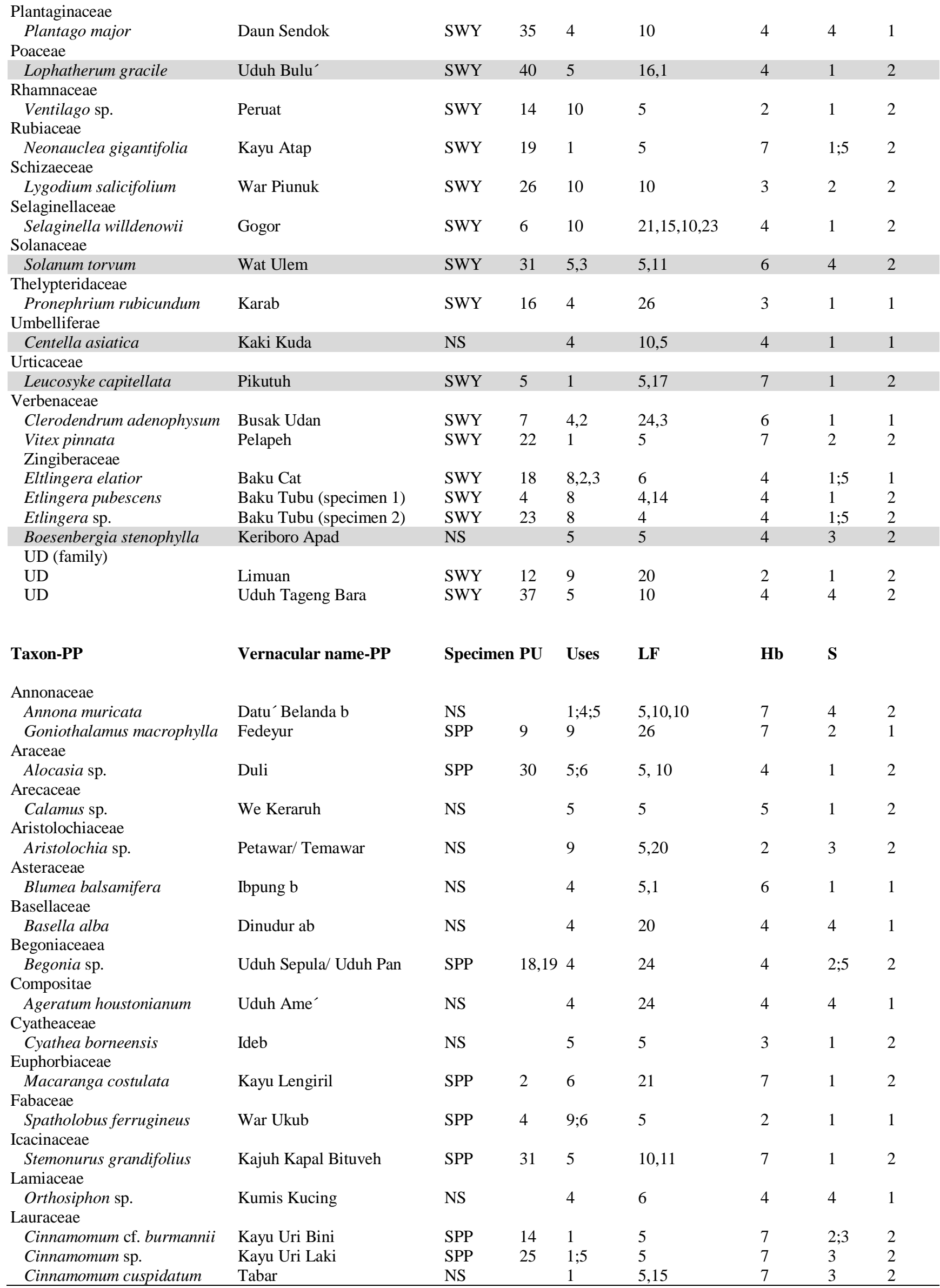


Linaceae

\begin{tabular}{|c|c|c|c|c|c|c|c|c|}
\hline $\begin{array}{l}\text { Indorouchera griffithii } \\
\text { Loganiaceae }\end{array}$ & War Beker & SPP & 21 & 9 & 5 & 2 & 3 & 1 \\
\hline Fagraea racemosa & Kayu Yureng & SPP & 26 & $5 ; 4$ & 5,21 & 7 & 1 & 2 \\
\hline \multicolumn{9}{|l|}{ Malvaceae } \\
\hline Theobroma cacao & Ya’un Koko & NS & & 4 & 4 & 7 & 4 & 1 \\
\hline \multicolumn{9}{|l|}{ Melastomataceae } \\
\hline Melastoma malabathricum & Si'yang & NS & & $5 ; 4 ; 2 ; 8$ & $5,24,1$ & 6 & 1 & 2 \\
\hline UD & War Seruyung & SPP & 24 & 9 & 5 & 2 & 3 & 1 \\
\hline \multicolumn{9}{|l|}{ Meliaceae } \\
\hline Lansium domesticum & Kayu Lingat & SPP & 8 & $1 ; 7$ & 10 & 7 & 2 & 2 \\
\hline \multicolumn{9}{|l|}{ Menispermaceae } \\
\hline Coscinium penestratum & War Babas & SPP & 6 & 9 & $10,5,24,23$ & 2 & $1 ; 5$ & 1 \\
\hline Tinomiscium petiolare & War Birar (poisonous) & SPP & 10 & 9 & 23 & 2 & 5 & 1 \\
\hline \multicolumn{9}{|l|}{ Moraceae } \\
\hline Ficus pubinervis & Kayu Nawe & NS & & 6 & 24 & 7 & 2 & 2 \\
\hline Ficus sp. & Tad Al / Teratat Lal & SPP & 12 & $5 ; 9 ; 4$ & 10,5 & 2 & $2 ; 3$ & 2 \\
\hline \multicolumn{9}{|l|}{ Musaceae } \\
\hline Musa sp. & Ya'ung & NS & & 9 & 22 & 4 & 4 & 2 \\
\hline \multicolumn{9}{|l|}{ Myrsinaceae } \\
\hline Maesa ramentacea & Kayu Pentenab & SPP & 5 & $4 ; 8$ & 5,6 & 2 & 1 & 1 \\
\hline Embelia sp. & War Ilang & SPP & 3 & 5 & & 2 & 1 & 1 \\
\hline \multicolumn{9}{|l|}{ Myrtaceae } \\
\hline Psidium guajava & $\mathrm{Va}^{\prime} \mathrm{Ibuh}$ & NS & & $4 ; 8$ & 5 & 7 & 4 & 2 \\
\hline \multicolumn{9}{|l|}{ Poaceae } \\
\hline Lophatherum gracile & Uduh Bulu' & SPP & 16 & 5 & 6,10 & 4 & $2 ; 3$ & 2 \\
\hline Paspalum sp. & Uduh Kerbau & SPP & 17 & 6 & 24,10 & 4 & 1 & 2 \\
\hline \multicolumn{9}{|l|}{ Polypodiaceae } \\
\hline Crypsinopsis subfasciatus & Uduh Putut Urat & SPP & 29 & 10 & 24,8 & 4 & $1 ; 5$ & 2 \\
\hline \multicolumn{9}{|l|}{ Rubiaceae } \\
\hline Uncaria lanosa & War Kelawit & SPP & 13 & 9 & 5 & 2 & $2 ; 3$ & 1 \\
\hline \multicolumn{9}{|l|}{ Simaroubaceae } \\
\hline Eurycoma longifolia & Pasak Bumi & SPP & 23 & 5 & 4,5 & 6 & 3 & 2 \\
\hline \multicolumn{9}{|l|}{ Solanaceae } \\
\hline Solanum torvum & Wat Ulem & NS & & $5 ; 3$ & 10,4 & 6 & 4 & 1 \\
\hline \multicolumn{9}{|l|}{ Umbelliferae } \\
\hline Eryngium foetidum & Sop Hutan & SPP & 27 & $4 ; 9$ & 4 & 4 & $1 ; 5$ & 1 \\
\hline Centella asiatica & Uduh Pelali & SPP & 28 & 4 & 10,5 & 4 & $1 ; 5$ & 1 \\
\hline \multicolumn{9}{|l|}{ Urticaceae } \\
\hline Leucosyke capitellata & Pikutuh & NS & & $5 ; 1$ & 5 & 7 & 2 & 2 \\
\hline \multicolumn{9}{|l|}{ Zingiberaceae } \\
\hline $\begin{array}{l}\text { Boesenbergia stenophylla } \\
\text { UD (family) }\end{array}$ & Teboro Apad Betina & SPP & 22 & 9 & 10,5 & 4 & 3 & 2 \\
\hline Charcoal & Arang Kayu Api & NS & & & 10 & 7 & & \\
\hline UD & Rumput Sepula Air & SPP & 11 & 10 & 10 & 1 & $2 ; 3$ & 2 \\
\hline
\end{tabular}

Note: Specimen (All voucher specimens deposited in Herbarium Wanariset, Samboja, East Kalimantan, Indonesia): 1 SWY: Wa' Yagung; 2 SPP: Pa Padi; 3 NS: No Specimen. LF (life form) is the life form of the medicinal plant species: 1 Aquatic plant; 2 Vine; 3 Fern; 4 Herb; 5 Rattan; 6 Shrub; 7 Tree. Hb (habitat) is the habitat based on the indigenous land-use system of the medicinal plants found during transect walk: 1 Young secondary forest (Amug Dari); 2 Old secondary forest (Amug Kara); 3 Primary forest (Pulung Kara/Pulung Kura); 4 Forest garden (Lati'); 5 Ricefield (Ba'). S (sustainability) is the sustainability probability for the plants by extracting method: 1 more concern; 2 less concern. Common medicinal plant species identification using photographs from the field and literature from Sangat et al. (2000), Matius et al. (2003), Yuliarti (2008), and the voucher specimen collection at Herbarium Samboja. UD is undetermined. PU (part used) is plant part used: 1 Bark; 2 Flower; 3 Fruit; 4 Leaf; 5 Root; 6 Sap; 7 Seed; 8 Shoot; 9 Stem; 10 Whole Plant. U (use) is the use of the plants as medicine (adapted using Level 2 ailment category by Cook (1995):

1. unspecified medicinal disorder

2. abnormalities

3. blood system disorder

4. circulatory system disorder

5. digestive system disorder

6. endocrine system disorder

7. genitourinary system disorder

8. ill-defined symptoms

9. immune system disorder
10. infections/infestations

11. inflammation

12. injuries

13. mental disorder

14. metabolic system disorder

15. muscular-skeletal system disorder

16. neoplasms

17. nervous system disorder

18. nutritional disorder
19. pain

20. poisonings

21. pregnancy/birth/puerperium

22. respiratory system disorder

23 . sensory system disorder

24. skin/subcutaneous cellular tissue disorder

25. veterinary

26. cultural/repellent 
Table 2. Knowledge and use in Wa' Yagung and Pa' Padi, Kayan Mentarang National Park (KMNP), North Kalimantan, Indonesia

\begin{tabular}{|c|c|c|c|c|c|c|c|c|c|c|c|}
\hline \multirow{2}{*}{$\begin{array}{l}\text { Taxon-WY } \\
\end{array}$} & \multirow{2}{*}{ Vernacular name-WY } & \multicolumn{5}{|c|}{ Value of knowledge (VK)-WY } & \multicolumn{5}{|c|}{ Value of use (VU)-WY } \\
\hline & & G1 & G2 & G3 & G4 & Mean & G1 & G2 & G3 & G4 & Mean \\
\hline \multicolumn{12}{|l|}{ Apocynaceae } \\
\hline Alstonia scholaris & Angeh & 10 & 9 & 8 & 9 & 0.97 & 6 & 8 & 5 & 8 & 0.73 \\
\hline \multicolumn{12}{|l|}{ Araceae } \\
\hline Acorus calamus & Keriboro Tuu & 2 & 9 & 5 & 7 & 0.62 & 2 & 1 & 2 & 3 & 0.22 \\
\hline \multicolumn{12}{|l|}{ Arecaceae } \\
\hline Calamus sp. & We Keraruh & 2 & 8 & 5 & 8 & 0.62 & 0 & 1 & 2 & 5 & 0.22 \\
\hline \multicolumn{12}{|l|}{ Aristolochiaceae } \\
\hline Aristolochia sp. & Temawar & 10 & 10 & 7 & 9 & 0.97 & 4 & 6 & 7 & 8 & 0.68 \\
\hline \multicolumn{12}{|l|}{ Asclepiadaceae } \\
\hline Hoya sp. & War Perata & 0 & 2 & 0 & 2 & 0.11 & 1 & 3 & 2 & 2 & 0.22 \\
\hline \multicolumn{12}{|l|}{ Balsaminaceae } \\
\hline \multicolumn{12}{|l|}{ Basellaceae } \\
\hline Basella alba & Dinudur ab & 8 & 9 & 8 & 6 & 0.84 & 1 & 0 & 1 & 3 & 0.14 \\
\hline Blechnaceae & & & & & & & & & & & \\
\hline Blechnum indicum & Pau Kelasi & 0 & 3 & 4 & 7 & 0.38 & 0 & 1 & 2 & 1 & 0.11 \\
\hline Bromeliaceae & & & & & & & & & & & \\
\hline Ananas sp. & Nanas ab & 1 & 3 & 0 & 1 & 0.14 & 1 & 1 & 1 & 2 & 0.14 \\
\hline Caricaceae & & & & & & & & & & & \\
\hline Carica papaya & Pepaya ab & 5 & 8 & 7 & 6 & 0.70 & 3 & 1 & 5 & 3 & 0.32 \\
\hline Compositae & & & & & & & & & & & \\
\hline Ageratum houstonianum & Uduh Ame' & 4 & 9 & 6 & 5 & 0.65 & 1 & 2 & 4 & 2 & 0.24 \\
\hline Convolvulaceae & & & & & & & & & & & \\
\hline Erycibe sp. & Lab Menipal & 5 & 7 & 7 & 9 & 0.76 & 2 & 3 & 5 & 3 & 0.35 \\
\hline Crassulaceae & & & & & & & & & & & \\
\hline Kalanchoe pinnata & Daun Lamak/Cocor Bebek a & 10 & 10 & 8 & 8 & 0.97 & 1 & 3 & 3 & 3 & 0.27 \\
\hline Cyatheaceae & & & & & & & & & & & \\
\hline Cyathea borneensis & Ideb & 1 & 4 & 5 & 7 & 0.46 & 0 & 0 & 2 & 0 & 0.05 \\
\hline Fabaceae & & & & & & & & & & & \\
\hline Archidendron clypearia & Kayu Keraruh & 1 & 2 & 2 & 7 & 0.32 & 0 & 0 & 0 & 0 & 0.00 \\
\hline Flacourtiaceae & & & & & & & & & & & \\
\hline Flacourtia rukam & Tateh & 3 & 4 & 3 & 8 & 0.49 & 1 & 1 & 1 & 3 & 0.16 \\
\hline Lamiaceae & & & & & & & & & & & \\
\hline Orthosiphon sp. & Kumis Kucing ab & 10 & 10 & 8 & 9 & 1.00 & 3 & 2 & 3 & 2 & 0.27 \\
\hline Lauraceae & & & & & & & & & & & \\
\hline Cinnamomum cuspidatum & Tabar & 8 & 10 & 7 & 9 & 0.92 & 4 & 9 & 8 & 7 & 0.76 \\
\hline Liliaceae & & & & & & & & & & & \\
\hline Smilax sp. & Uduh Bila'Serei' & 1 & 6 & 3 & 8 & 0.49 & 0 & 0 & 2 & 1 & 0.08 \\
\hline Magnoliaceae & & & & & & & & & & & \\
\hline Elmerillia tsiampacca & Merebung & 6 & 5 & 8 & 9 & 0.76 & 0 & 2 & 1 & 0 & 0.08 \\
\hline Malvaceae & & & & & & & & & & & \\
\hline Sida rhomboidea & Lab Uri' & 1 & 3 & 3 & 2 & 0.24 & 0 & 0 & 1 & 2 & 0.08 \\
\hline Melastomataceae & & & & & & & & & & & \\
\hline Melastoma malabathricum & Sikeli' & 10 & 10 & 8 & 9 & 1.00 & 1 & 4 & 3 & 4 & 0.32 \\
\hline Diplectria sp. & Uduh Lab Batek & 9 & 7 & 3 & 5 & 0.65 & 0 & 0 & 2 & 0 & 0.05 \\
\hline UD & Rumput WY & 0 & 0 & 0 & 0 & 0.00 & 0 & 0 & 0 & 0 & 0.00 \\
\hline Meliaceae & & & & & & & & & & & \\
\hline Lansium domesticum & Kayu Lingat/Langsat a & 2 & 6 & 4 & 8 & 0.54 & 0 & 1 & 2 & 3 & 0.16 \\
\hline Menispermaceae & & & & & & & & & & & \\
\hline Tinospora crispa & Babas/Brotowali ab & 10 & 10 & 8 & 9 & 1.00 & 0 & 1 & 0 & 5 & 0.16 \\
\hline Tinospora sp. & Tutud Ada' & 7 & 10 & 6 & 9 & 0.86 & 2 & 2 & 5 & 3 & 0.32 \\
\hline Fibraurea tinctoria & War Birar & 6 & 8 & 5 & 9 & 0.76 & 0 & 1 & 1 & 1 & 0.08 \\
\hline Myrsinaceae & & & & & & & & & & & \\
\hline Maesa ramentacea & Uduh Lepen & 1 & 2 & 3 & 6 & 0.32 & 0 & 0 & 0 & 0 & 0.00 \\
\hline Myrtaceae & & & & & & & & & & & \\
\hline Psidium guajava & OKK/Bua' Libuh b & 9 & 9 & 6 & 9 & 0.89 & 1 & 1 & 1 & 5 & 0.22 \\
\hline Nephrolepidaceae & & & & & & & & & & & \\
\hline Nephrolepis multiflora & Pau Bulat & 2 & 3 & 3 & 7 & 0.41 & 0 & 0 & 2 & 0 & 0.05 \\
\hline Phyllanthaceae & & & & & & & & & & & \\
\hline Phyllanthus urinaria & Uduh Babeh Anak/Meniran & 10 & 10 & 6 & 9 & 0.95 & 2 & 3 & 5 & 5 & 0.41 \\
\hline Piperaceae & & & & & & & & & & & \\
\hline Piper sp. & Daun Buyu' & 8 & 6 & 7 & 9 & 0.81 & 0 & 1 & 2 & 3 & 0.16 \\
\hline Piper sp. & War Siri & 1 & 4 & 2 & 4 & 0.30 & 0 & 2 & 1 & 0 & 0.08 \\
\hline
\end{tabular}




\begin{tabular}{|c|c|c|c|c|c|c|c|c|c|c|c|}
\hline \multicolumn{12}{|l|}{ Plantaginaceae } \\
\hline Plantago major & Daun Sendok & 10 & 10 & 8 & 9 & 1.00 & 7 & 3 & 4 & 4 & 0.49 \\
\hline \multicolumn{12}{|l|}{ Poaceae } \\
\hline Lophatherum gracile & Uduh Bulu’ & 2 & 8 & 3 & 6 & 0.51 & 0 & 0 & 1 & 0 & 0.03 \\
\hline \multicolumn{12}{|l|}{ Rhamnaceae } \\
\hline Ventilago sp. & Peruat & 0 & 4 & 4 & 8 & 0.43 & 0 & 3 & 1 & 2 & 0.16 \\
\hline \multicolumn{12}{|l|}{ Rubiaceae } \\
\hline Neonauclea gigantifolia & Kayu Atap & 4 & 4 & 3 & 6 & 0.46 & 0 & 1 & 1 & 3 & 0.14 \\
\hline \multicolumn{12}{|l|}{ Schizaeceae } \\
\hline Lygodium salicifolium & War Piunuk & 1 & 2 & 2 & 4 & 0.24 & 0 & 1 & 0 & 3 & 0.11 \\
\hline \multicolumn{12}{|l|}{ Selaginellaceae } \\
\hline Selaginella willdenowii & Gogor & 2 & 5 & 4 & 8 & 0.51 & 0 & 1 & 3 & 1 & 0.14 \\
\hline \multicolumn{12}{|l|}{ Solanaceae } \\
\hline \multicolumn{11}{|l|}{ Thelypteridaceae } & 0.27 \\
\hline Pronephrium rubicundum & Karab & 10 & 8 & 6 & 8 & 0.86 & 0 & 0 & 2 & 1 & 0.08 \\
\hline \multicolumn{12}{|l|}{ Umbelliferae } \\
\hline Centella asiatica & Kaki Kuda & 1 & 3 & 4 & 9 & 0.46 & 0 & 1 & 3 & 2 & 0.16 \\
\hline \multicolumn{12}{|l|}{ Urticaceae } \\
\hline Leucosyke capitellata & Pikutuh & 1 & 3 & 5 & 8 & 0.46 & 0 & 1 & 2 & 2 & 0.14 \\
\hline \multicolumn{12}{|l|}{ Verbenaceae } \\
\hline Clerodendrum adenophysum & Busak Udan & 1 & 1 & 7 & 9 & 0.49 & 0 & 0 & 0 & 0 & 0.00 \\
\hline Vitex pinnata & Pelapeh & 3 & 6 & 5 & 5 & 0.51 & 0 & 1 & 1 & 1 & 0.08 \\
\hline \multicolumn{12}{|l|}{ Zingiberaceae } \\
\hline Etlingera elatior & Baku' Cat & 8 & 8 & 5 & 6 & 0.73 & 1 & 2 & 4 & 2 & 0.24 \\
\hline Etlingera pubescens & Baku’Tubu & 1 & 4 & 3 & 7 & 0.41 & 0 & 1 & 4 & 3 & 0.22 \\
\hline $\begin{array}{l}\text { Boesenbergia stenophylla } \\
\text { UD (family) }\end{array}$ & Keriboro Apad & 0 & 2 & 4 & 8 & 0.38 & 0 & 1 & 1 & 2 & 0.11 \\
\hline UD & Limuan & 1 & 0 & 3 & 2 & 0.16 & 0 & 0 & 1 & 3 & 0.11 \\
\hline UD & Uduh Tageng Bara & 1 & 4 & 4 & 3 & 0.32 & 0 & 1 & 3 & 1 & 0.14 \\
\hline Taxon-PP & Vernacular name-PP & Val & e of & snow & edge & (K)-PP & & Valu & of $\mathbf{u}$ & (VI & -PP \\
\hline & & G5 & G6 & G7 & G8 & Mean & G5 & G6 & G7 & G8 & Mean \\
\hline Annonaceae & & & & & & & & & & & \\
\hline Annona muricata & Datu’ Belanda & 1 & 1 & 9 & 7 & 0.60 & 0 & 1 & 1 & 6 & 0.27 \\
\hline Goniothalamus macrophylla & Fedeyur & 1 & 4 & 10 & 10 & 0.83 & 3 & 2 & 0 & 3 & 0.27 \\
\hline Araceae & & & & & & & & & & & \\
\hline Alocasia sp. & Duli' & 0 & 1 & 7 & 8 & 0.53 & 0 & 0 & 5 & 3 & 0.27 \\
\hline Arecaceae & & & & & & & & & & & \\
\hline Calamus sp. & We Keraruh & 2 & 5 & 10 & 10 & 0.90 & 0 & 1 & 7 & 6 & 0.47 \\
\hline Aristolochiaceae & & & & & & & & & & & \\
\hline Aristolochia sp. & Petawar & 4 & 4 & 10 & 9 & 0.90 & 5 & 3 & 10 & 10 & 0.93 \\
\hline Asteraceae & & & & & & & & & & & \\
\hline Blumea balsamifera & Ibpung & 1 & 1 & 1 & 5 & 0.27 & 1 & 1 & 1 & 5 & 0.27 \\
\hline Basellaceae & & & & & & & & & & & \\
\hline Basella alba & Dinudur ab & 1 & 3 & 4 & 9 & 0.57 & 2 & 2 & 3 & 4 & 0.37 \\
\hline Begoniaceae & & & & & & & & & & & \\
\hline Begonia sp. & Uduh Pan & 3 & 4 & 8 & 9 & 0.80 & 0 & 2 & 5 & 6 & 0.43 \\
\hline Compositae & & & & & & & & & & & \\
\hline Ageratum houstonianum & Uduh Ame' & 0 & 2 & 2 & 6 & 0.33 & 1 & 1 & 1 & 0 & 0.10 \\
\hline Cyatheaceae & & & & & & & & & & & \\
\hline Cyathea borneensis & Ideb & 1 & 2 & 7 & 5 & 0.50 & 0 & 1 & 1 & 1 & 0.10 \\
\hline Euphorbiaceae & & & & & & & & & & & \\
\hline Macaranga costulata & Kayu Lengiril & 3 & 3 & 9 & 7 & 0.73 & 0 & 0 & 6 & 1 & 0.23 \\
\hline Fabaceae & & & & & & & & & & & \\
\hline Spatholobus ferrugineus & War Ukub & 0 & 1 & 10 & 10 & 0.70 & 0 & 0 & 4 & 3 & 0.23 \\
\hline Icacinaceae & & & & & & & & & & & \\
\hline Stemonurus grandifolius & Kajuh Kapal Bituveh & 1 & 3 & 3 & 6 & 0.43 & 0 & 1 & 0 & 2 & 0.10 \\
\hline Lamiaceae & & & & & & & & & & & \\
\hline Orthosiphon sp. & Kumis Kucing ab & 5 & 5 & 8 & 10 & 0.93 & 3 & 2 & 4 & 5 & 0.47 \\
\hline Lauraceae & & & & & & & & & & & \\
\hline Cinnamomum cf.burmannii & Kayu Uri’ (Bini) & 1 & 2 & 7 & 9 & 0.63 & 0 & 1 & 3 & 4 & 0.27 \\
\hline Cinnaтотит сиspidatum & Tabar & 5 & 5 & 10 & 10 & 1.00 & 4 & 1 & 9 & 8 & 0.73 \\
\hline Linaceae & & & & & & & & & & & \\
\hline $\begin{array}{l}\text { Indorouchera griffithii } \\
\text { Loganiaceae }\end{array}$ & War Beker & 0 & 0 & 9 & 10 & 0.63 & 0 & 0 & 7 & 4 & 0.37 \\
\hline
\end{tabular}




\begin{tabular}{|c|c|c|c|c|c|c|c|c|c|c|c|}
\hline Fagraea racemosa & Kayu Yureng & 3 & 5 & 9 & 10 & 0.90 & 1 & 2 & 6 & 4 & 0.43 \\
\hline \multicolumn{12}{|l|}{ Malvaceae } \\
\hline Theobroma cacao & Yáun Koko b & 4 & 5 & 8 & 8 & 0.83 & 0 & 1 & 1 & 2 & 0.13 \\
\hline \multicolumn{12}{|l|}{ Melastomataceae } \\
\hline Melastoma malabathricum & Si'yang & 5 & 5 & 10 & 10 & 1.00 & 4 & 4 & 8 & 7 & 0.77 \\
\hline UD & War Seruyung & 5 & 5 & 7 & 10 & 0.90 & 1 & 3 & 4 & 10 & 0.60 \\
\hline \multicolumn{12}{|l|}{ Meliaceae } \\
\hline Lansium domesticum & Kayu Langsat a & 1 & 3 & 8 & 10 & 0.73 & 1 & 1 & 2 & 5 & 0.30 \\
\hline \multicolumn{12}{|l|}{ Menispermaceae } \\
\hline Coscinium penestratum & War Babas & 1 & 3 & 10 & 10 & 0.80 & 2 & 2 & 6 & 8 & 0.60 \\
\hline Tinomiscium petiolare & War Birar & 0 & 0 & 8 & 10 & 0.60 & 0 & 0 & 3 & 3 & 0.20 \\
\hline \multicolumn{12}{|l|}{ Moraceae } \\
\hline Ficus pubinervis & Kayu Nawe & 2 & 2 & 4 & 9 & 0.57 & 0 & 0 & 2 & 2 & 0.13 \\
\hline Ficus sp. & Kayu Teratat Lal & 1 & 2 & 1 & 4 & 0.27 & 0 & 0 & 1 & 4 & 0.17 \\
\hline \multicolumn{12}{|l|}{ Musaceae } \\
\hline Musa sp. & Ya'ung b & 2 & 1 & 2 & 4 & 0.30 & 0 & 0 & 0 & 2 & 0.07 \\
\hline \multicolumn{12}{|l|}{ Myrsinaceae } \\
\hline Embelia sp. & War Ilang & 0 & 0 & 8 & 5 & 0.43 & 0 & 1 & 2 & 2 & 0.17 \\
\hline Maesa ramentaceae & Kayu Pentenab & 0 & 2 & 3 & 8 & 0.43 & 0 & 0 & 3 & 5 & 0.27 \\
\hline \multicolumn{12}{|l|}{ Myrtaceae } \\
\hline Psidium guajava & $\mathrm{Va}^{\prime} \mathrm{Ibuh} \mathrm{b}$ & 5 & 4 & 10 & 10 & 0.97 & 4 & 3 & 7 & 6 & 0.67 \\
\hline \multicolumn{12}{|l|}{ Poaceae } \\
\hline Lophatherum gracile & Uduh Bulu’ & 2 & 4 & 7 & 10 & 0.77 & 0 & 1 & 2 & 4 & 0.23 \\
\hline Paspalum sp. & Uduh Kerbau & 5 & 4 & 9 & 10 & 0.93 & 2 & 2 & 4 & 3 & 0.37 \\
\hline \multicolumn{12}{|l|}{ Polypodiaceae } \\
\hline Crypsinopsis subfaciatus & Uduh Putut Urat & 1 & 2 & 1 & 4 & 0.27 & 0 & 0 & 0 & 2 & 0.07 \\
\hline \multicolumn{12}{|l|}{ Rubiaceae } \\
\hline Uncaria lanosa & War Kelawit & 0 & 0 & 5 & 7 & 0.40 & 0 & 0 & 2 & 2 & 0.13 \\
\hline \multicolumn{12}{|l|}{ Simaroubaceae } \\
\hline Eurycoma longifolia & Pasak Bumi a & 4 & 5 & 6 & 8 & 0.77 & 0 & 2 & 0 & 4 & 0.20 \\
\hline \multicolumn{12}{|l|}{ Solanaceae } \\
\hline Solanum torvum & Wat Ulem b & 5 & 5 & 9 & 8 & 0.90 & 1 & 0 & 3 & 5 & 0.30 \\
\hline \multicolumn{12}{|l|}{ Umbelliferae } \\
\hline Eryngium foetidum & Sop Hutan & 1 & 1 & 1 & 7 & 0.33 & 0 & 1 & 0 & 0 & 0.03 \\
\hline Centella asiatica & Uduh Pelali & 5 & 5 & 4 & 10 & 0.80 & 1 & 3 & 3 & 6 & 0.43 \\
\hline \multicolumn{12}{|l|}{ Urticaceae } \\
\hline Leucosyke capitellata & Pikutuh & 0 & 0 & 2 & 5 & 0.23 & 0 & 0 & 1 & 1 & 0.07 \\
\hline \multicolumn{12}{|l|}{ Zingiberaceae } \\
\hline $\begin{array}{l}\text { Boesenbergia stenophylla } \\
\text { UD (family) }\end{array}$ & Teboro Abpad & 0 & 2 & 6 & 10 & 0.60 & 0 & 0 & 2 & 0 & 0.07 \\
\hline UD & Rumput Sepula' Air & 1 & 1 & 6 & 9 & 0.57 & 1 & 0 & 2 & 2 & 0.17 \\
\hline- & Arang Kayu Api a & 2 & 1 & 2 & 8 & 0.43 & 0 & 1 & 3 & 3 & 0.23 \\
\hline
\end{tabular}

Notes: The highlighted ones are the three highest mean value for knowledge and use. The bold ones found in both villages. Respondents: G1 (Wa' Yagung: 10); G5 (Pa' Padi: 5): respondents of younger women, G2 (Wa' Yagung: 10); G6 (Pa' Padi: 5): respondents of older women, G3 (Wa' Yagung: 8); G7 (Pa' Padi: 10): respondents of younger men, G4 (Wa' Yagung: 9); G8 (Pa' Padi: 10): respondents of older men. a is the common Indonesian plants name and spoken in Wa' Yagung (Table 2-1) and Pa' Padi (Table 22). $\mathrm{b}$ is common plants species confirmed to Herbarium Samboja using images taken during fieldwork, also literature by Sangat et al. (2000), Matius et al. (2003), and Yuliarti (2008). UD is undetermined. OKK is Obat Kuta'Katei'

Table 3. Number of medicinal plant species found in habitat

\begin{tabular}{|c|c|c|c|c|c|}
\hline \multirow{2}{*}{ Local land use } & \multirow{2}{*}{ Descriptions } & \multicolumn{2}{|c|}{ Wa' Yagung } & \multicolumn{2}{|c|}{ Pa'Padi } \\
\hline & & $\#$ & $\%$ & $\#$ & $\%$ \\
\hline $\begin{array}{l}\text { Amug Dari } \\
\text { (young secondary forest) }\end{array}$ & $\begin{array}{l}\text { Fallow }<10 \text { years dominated by light-demanding and pioneer } \\
\text { species, higher in density, at a lower elevation, and at the vicinity of } \\
\text { the village }\end{array}$ & 32 & 50.79 & 18 & 30.51 \\
\hline $\begin{array}{l}\text { Amug Kara } \\
\text { (old secondary forest) }\end{array}$ & $\begin{array}{l}\text { Fallow }>10 \text { years, bigger in dbh, at lower to a higher elevation, and } \\
\text { at the vicinity of the village }\end{array}$ & 7 & 11.11 & 14 & 23.73 \\
\hline $\begin{array}{l}\text { Pulung Kara (WY)/P. Kura } \\
(\mathrm{PP}) \\
\text { (primary forest) }\end{array}$ & $\begin{array}{l}\text { Never been disturbed, diverse in structure, tree species, at a higher } \\
\text { elevation, steep slope, at some distance from the village }\end{array}$ & 4 & 6.35 & 13 & 22.03 \\
\hline $\begin{array}{l}\text { Lati } \\
\text { (forest garden) }\end{array}$ & $\begin{array}{l}\text { Annual crops for another edible food source situated surround the } \\
\text { village area }\end{array}$ & 16 & 25.40 & 7 & 11.86 \\
\hline $\begin{array}{l}\mathrm{Ba}^{\prime} \\
\text { (rice field) }\end{array}$ & $\begin{array}{l}\text { Rice crops for a staple food source, situated surround the village } \\
\text { area }\end{array}$ & 4 & 6.35 & 7 & 11.86 \\
\hline
\end{tabular}


Some exotic species also occurred in both villages such as Carica papaya, Orthosiphon sp., Psidium guajava, or Kalanchoe pinnata that widely acknowledged as medicinal plants based on the experience of Mueller and Mechler (2005) in the Democratic Republic of Congo. The villagers in Wa' Yagung said they almost never cultivate the medicinal plant's species around their settlement, rice field, and forest garden, except one household whose husband grew Tinospora crispa for his own hypertension therapy. In Pa'Padi, several villagers mentioned the reason for the cultivation of some exotic species at home yard on purpose of medicine and diet source. Such as Plantago major originated from northern Europe and Central Asia (Velasco-Lezama et al. 2006) but found in both villages explains the characteristics of Krayan area of mostly montane forests which resembles temperature and altitude of forest type in Northern Europe and Central Asia. The temperature will be lower $5^{\circ} \mathrm{C}$ for every $1,000 \mathrm{~m}$ altitude (WWF 2007). This statement also supported by the presence of conifers (which usually associated with colder climates of the northern hemisphere) in the tropical rainforest of Borneo, which most of them occur at the higher elevation only in Krayan area of KMNP (Wulffrat 2005).

\section{Life form and plant part}

Herbs species served as the most usable for medicine in Wa' Yagung for 17 (33\%), followed by vines, trees, ferns, shrubs, and rattan respectively (Table 4). On the other hand, in Pa'Padi, trees were identified as the most usable for 13 species (30.95\%), followed herbs, vines, shrubs, and one each of fern, rattan, and aquatic plant (Table 4). Species of herbs supplied as dishes, in particular, the shoots, flowers, fruits, and inner stems, while their leaves used as food wrapper or thatches of the temporary shed. Herbs are categorized as an understory, therefore this supports the most used plants by the villagers which mostly in young secondary forest. People tend to use plants available at their vicinity. The plant part which villagers used most as medicines in both villages was leaf (Table 4), and some had to mix with other species or plant part in precautionary principles in preparation and application. Therefore, people sometimes confirmed the local healer for some ailments or they asked for the medicine compounds prepared by the local healer.

Table 4. Number of medicinal plant species based on life form

\begin{tabular}{lcccc}
\hline \multirow{2}{*}{ Life form } & \multicolumn{2}{c}{ Wa' Yagung } & \multicolumn{2}{c}{ Pa'Padi } \\
& $\#$ & $\mathbf{\%}$ & $\#$ & $\mathbf{\%}$ \\
\hline Aquatic plant & 0 & 0.00 & 1 & 2.38 \\
Vine & 13 & 25.49 & 10 & 23.81 \\
Fern & 6 & 11.76 & 1 & 2.38 \\
Herb & 16 & 33.33 & 11 & 26.19 \\
Rattan & 1 & 1.96 & 1 & 2.38 \\
Shrub & 5 & 9.80 & 5 & 11.90 \\
Tree & 9 & 17.65 & 13 & 30.95 \\
\hline
\end{tabular}

Table 5. Number of medicinal plant species based on the plant part

\begin{tabular}{lcccc}
\hline \multirow{2}{*}{ Plant part used } & \multicolumn{2}{c}{ Wa' Yagung } & \multicolumn{2}{c}{ Pa'Padi } \\
& $\#$ & $\mathbf{\%}$ & $\#$ & \% \\
\hline Bark & 9 & 12.86 & 6 & 9.09 \\
Flower & 3 & 4.29 & 1 & 1.52 \\
Fruit & 4 & 5.71 & 1 & 1.52 \\
Leaf & 16 & 22.86 & 16 & 24.24 \\
Root & 8 & 11.43 & 15 & 22.73 \\
Sap & 3 & 4.29 & 7 & 10.61 \\
Seed & 0 & 0.00 & 1 & 1.52 \\
Shoot & 10 & 14.29 & 3 & 4.55 \\
Stem & 12 & 17.14 & 14 & 21.21 \\
All parts & 5 & 7.14 & 2 & 3.03 \\
\hline
\end{tabular}
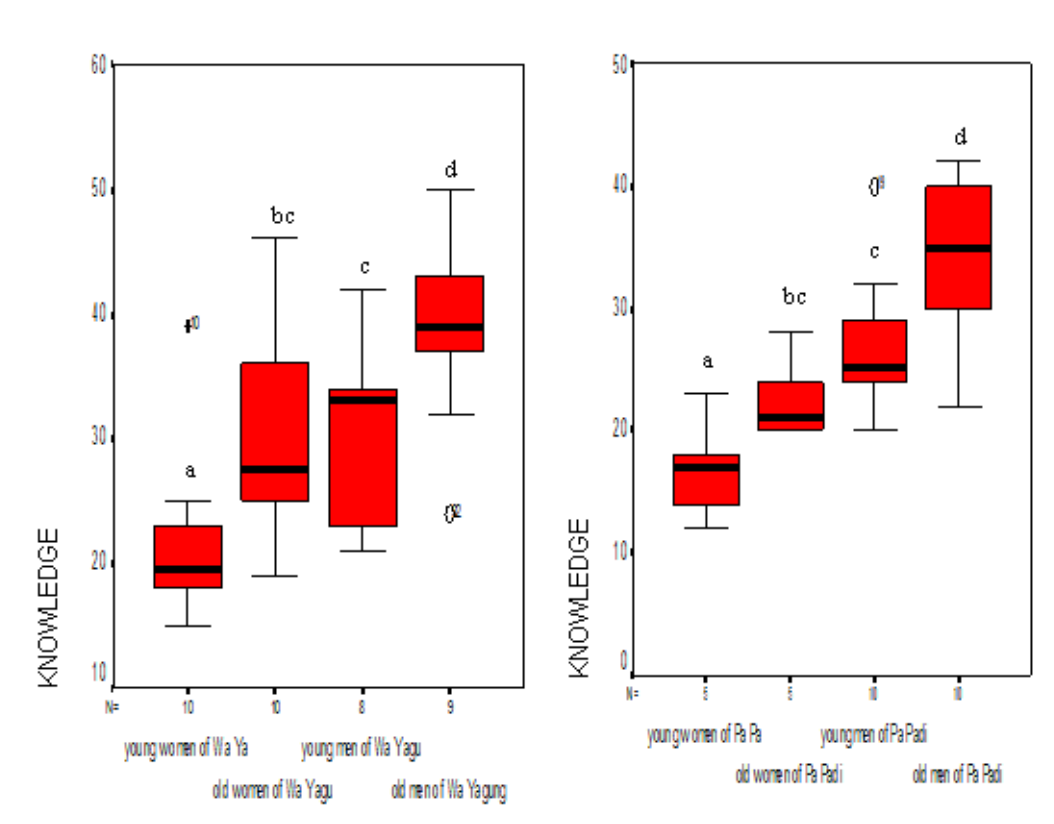

Figure 2. Knowledge in Wa' Yagung (left) and Pa' Padi (right), Kayan Mentarang National Park, North Kalimantan, Indonesia 
Leaves were the mostly plant part used for medicinal practice in Wa' Yagung and Pa'Padi. This could explain in this primary photosynthetic organ of the tree species (Harding et al. 2001), bioactive secondary plant products such alkaloids, simple phenols, and cyanogenic glycosides widely in angiosperms were found besides in roots and fruits (Larcher 1995). It would be a sustainable way as the characteristic of this aerial vegetative organ is ephemeral, quickly shed and their functions could be taken over by the stem (Cutler et al. 2008).

The most plant part used in Wa' Yagung were leaf for $16(22.86 \%)$, followed by the stem, shoot, bark, root, whole plant, fruit, and sap and flower, and no seed used (Table 5). While likewise in Pa'Padi for leaf $(24.24 \%)$, and root, stem, sap, bark, shoot, fruit, seed, and flower respectively (Table 5). Here, leaves as the most important part in photosynthesis for plants growth, have properties of medicine. This supported by a statement leaves used by pharmacognosists in palisade ratio to determine small leaf fragments in leaf powder products (Cutler et al. 2007).

The life form of herbs dominantly used as medicinal plants species in Wa'Yagung whilst in Pa'Padi dominated by tree species. The difference in preferences of using medicinal plants appeared more likely, as herbs were the second-highest number in Pa'Padi. Besides, during the transect walks in both villages, such important herbs of edible Etlingera were rarely seen in Pa'Padi but abundant in Wa' Yagung. Villagers in Wa' Yagung said flower buds and fruits of Etlingera were the most tasteful vegetables from the forest.

\section{Knowledge and use of medicinal plants by villagers}

There is a significant difference in knowledge and use of medicinal plants using Kruskal-Wallis test among four groups in Wa' Yagung with $\mathrm{p}=0.001$ for knowledge and $\mathrm{p}=0.040$ for use and $\mathrm{Pa}$ Padi with $\mathrm{p}=0.000$ for knowledge and $\mathrm{p}=0.006$ for use. This means the post hoc MannWhitney $U$ test run to compare between two groups in each village.

Based on data analysis, Figure 1 shows the significant difference was identical for knowledge in both villages which found in all groups excluding in older women to young men. Similarly to the range of a number of species recognized by villagers in both villages which lowest in young women and highest in old men group. Knowledge of medicinal plants showed in higher number in Wa' Yagung explains the remote situation of the village had attached people more to the forest. Knowledge of older men and older women in higher number in $\mathrm{Wa}$ ' Yagung reflected the longer experience leads to stronger influence to someone's life. As explained by Johannes (1989) that older people have better details of knowledge of local resources and virtually share good information occur in long time phases. The significant differences in comparing to all groups except old women compared to young men in both villages explained that old women were mostly responsible for the domestics and rice field, therefore, reduced the opportunity of the women to explore the plant's species in the forest. Nevertheless, they experienced things from looking after the family household and gathered information from spouses, relatives, neighbors, or the local healer in Wa' Yagung, while it possibly lower contact more likely occurred in Pa'Padi. Men are responsible for the external activity of a household and in the community such as hunting and gathering or collaborate in building the village's public facilities and their neighbors' houses. In Wa' Yagung, this work requires the men to reside in the forest for several days and sometimes women are involved though only in particular case. When the research accomplished in Wa' Yagung, villagers of men and women were staying in the forest to repair the broken wood bridges that connect their village to other villages. Men were also responsible for the rice field which they work in a group of several households managing the rice field, as women and men had shared different tasks when managing rice field, however, intensive hunting and gathering activities have nurtured them to the wildlife resources. Some villagers of young women were high school students who just finished the final exam at high school at Long Bawan, the district capital and visiting their parents during the data gathering. Possibly, the young age and living not in the village lowered the intensity of contact with forest, hence their knowledge and use of the medicinal plant's species were lower in number, moreover young age tend to have the healthier condition of physic.

Subsequently, for the use of medicinal plants, Figure 2 shows there were two significant differences in comparing young women to young men and young women to old men in Wa' Yagung. While, in Pa'Padi, the significant differences found similar in young women to young men, and young women to old men, also in old women compare to old men as well. While for the ranges of the number were similar in knowledge, they were different in use which the highest number found in young men in $\mathrm{Wa}$ ' Yagung and old men in $\mathrm{Pa}$ ' Padi, as stated in Figure 3. Higher of uses of medicinal plants in $\mathrm{Wa}^{\prime}$ Yagung than Pa'Padi had a positive correlation to knowledge. Significant differences in uses in Wa' Yagung demonstrated men group set higher belief to use medicinal plants particularly the old groups, which mostly proved to themselves the efficacies of medicinal properties in plant species from the forest. While between women and men inclined to more physical work of the latter to have a more important role in family and community. Old women generally stay at home and therefore tend to use medicinal plants in the vicinity of the village or taking concoction prepared by the local healer. Young men used more medicinal plants in Wa' Yagung, explained young men's responsibilities to take care of the family and elders. Most frequent ailments were in the digestive system and infestations, with women mostly focused on the medicinal plants around forest garden to treat diseases as the effect of physical work, digestive, puerperal, self-hygiene, and in children, while men mostly cared the ailments of physical work and nervous system (Table 1). 

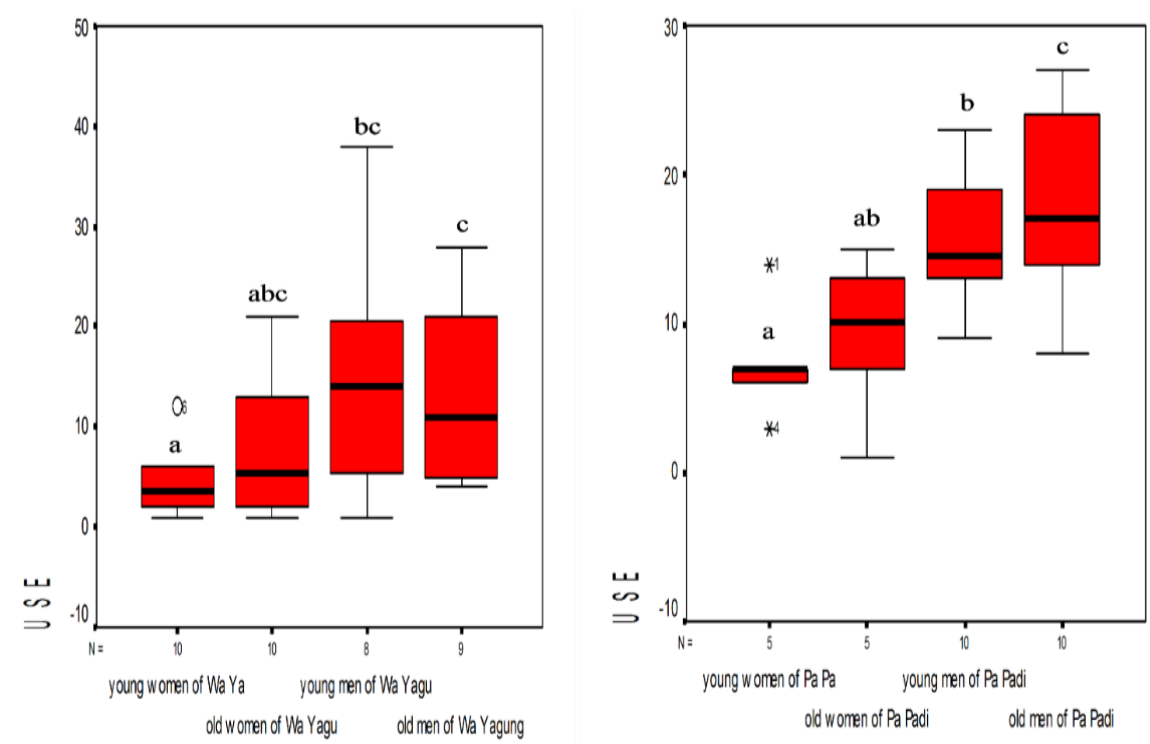

Figure 3. Use in Wa' Yagung (left) and Pa' Padi (right), Kayan Mentarang National Park, North Kalimantan, Indonesia

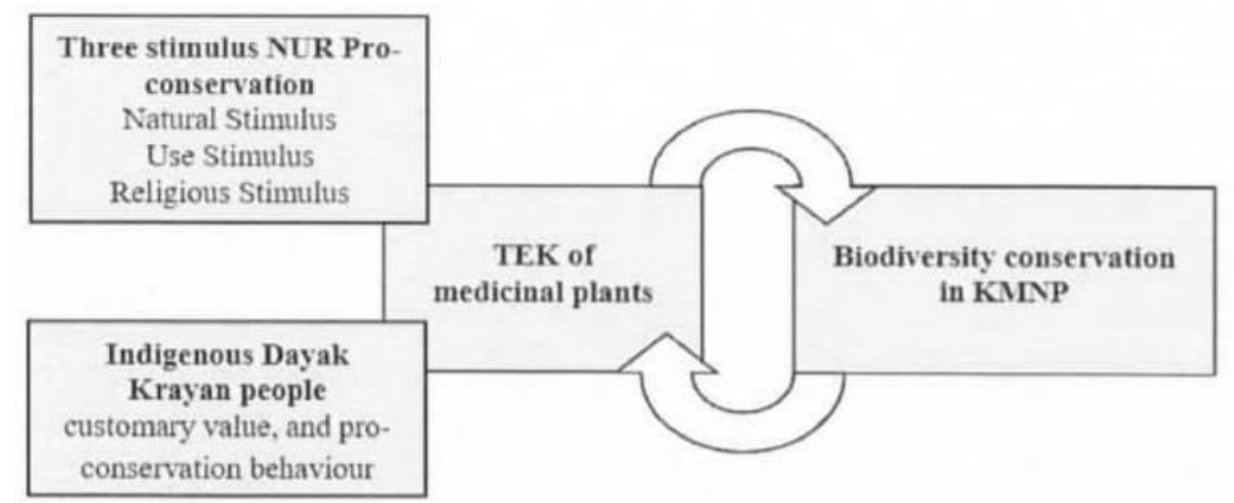

Figure 4. The linkage concept of traditional ecological knowledge of medicinal plants species and biodiversity conservation in Kayan Mentarang National Park (KMNP), North Kalimantan, Indonesia

Medicinal plants have an important role to the lives of villagers in both villages, particularly more to $\mathrm{Wa}$ ' Yagung as the remotest village and limited access to the public health centre in district capital consequently caused free and abundant natural resources in the forest become staple support, accompanying their belief on traditional medicine as the healthiest therapy. This situation had been occurred as typical in many tropical countries as described by Mueller and Mechler (2005) that in northeastern Congo, people depend on medicinal plants as national roads and provision of imported medicines restricted them from getting health services from the country. Local healer presence was also as motivating villagers to use medicinal plants here, which most households in Wa' Yagung stored at home concoction prepared by the local healer or dried medicinal plant parts, which happened very rarely in the households in Pa'Padi.

\section{The occurrence of the local important species in the habitat}

Tabar and Temawar in Wa' Yagung found in primary forest and Angeh in primary and young secondary forests. An older man in Wa' Yagung shared his opinion that strong good medicinal plants were usually very rare and difficult to find. While local healer and villagers in $\mathrm{Wa}$ Yagung informed that tree species of Tabar and Angeh used as timber for furniture and houses. The wood of Angeh (Alstonia scholaris) was the raw material of church's seats in Wa' Yagung', while Tabar described as a big tall tree and good timber. WCMC (1998) stated that the use of Alstonia scholaris as the most important source of pulai timber is a major threat of this species. The occurrence of the local important species in primary forest correlated to the characteristic of tree habit and medicinal efficacy (Sheldon and Balick 1995). Trees are K-strategists 
and rely on a high amount of energy sustained in each individual, therefore causes long vegetative phase and slow growth in population (Mosbrugger 1990). Added by Odum (1969) that trees need mature systems to grow to have more capacity to capture and sustain nutrients for rotation sequence in the system. The impact to tree species is bigger as according to Turner (2001), most trees occur at densities less than 10 mature individuals per hectare of forest and more in sparser population. Also supported by Fitter and Hay (2002), long life cycles of trees have caused a disadvantage in areas with disturbance, hence herbaceous vegetation will dominate. The rare occurrence of Temawar vines due to their characteristics, Schnitzer and Bongers (2002) explained lianas are a polyphyletic group of woody plants which has a strategy to grow to ascend to the canopy using other plants, and liana helps maintaining plant diversity in tropics after treefall gaps. Therefore, the occurrence of vines in the primary forest of Wa' Yagung and $\mathrm{Pa}$ 'Padi depends on the occurrence of trees, which mostly found in the primary forest and their architectures support the vine to grow ascendingly. The shrub of Si'yang (Melastoma malabathricum) is abundant in the accessible young secondary forest and around village Pa'Padi, and this situation has occurred since mid of the 40s in KerayanKelabit area as illustrated by Schneeberger (1945) where shrub vegetation in villages ruminated by water buffaloes, cattle, and goats, also scattered in open meadows, which give the landscape a parklike appearance.

\section{Medicinal plants and three stimuli NUR pro- conservation in Krayan}

Three stimuli NUR pro-conservation reviews traditional ecological knowledge of medicinal plants of Dayak Krayan people in supporting biodiversity conservation in KMNP. First, the natural stimulus can be observed from the unthreatened situation of biodiversity in KMNP. Villagers recognize the importance of the resilience of an ecosystem, and this is proved from their practices of sustainable agriculture system and extracting method of plant species intent to ensure the health and sustainability of the area and the species. During one of the transect walks when collecting Tabar (Cinnamomum cuspidatum) in $\mathrm{Wa}$ ' Yagung, the local healer took the remaining parts of the branch cut for the specimen and did not debark the tree to sustain this big tree. Leaves left to litter the forest floor. He also emphasized branches have the same therapeutical properties. Second, usefulness stimulus is apparent from almost all plants species useful to villagers in both villages in fulfillment of basic necessities of dishes, timber, and medicine without any cash to obtain those benefits from the forest. This explains people's strong dependability to their forest in this area. Lastly, third, religious stimulus observed acknowledgment of customary law by villagers to the present day. Also, a strong bond among villages showed how villagers believe good deeds in helping each other when a member of a household is sick. Local healers will never charge for the medicine, and other villagers help to collect the plants from the forest. When the situation gets worst for a villager, other villagers will roam the forest to find better medicine according to a recommendation from the local healer. Customary value in the Dayak people implemented into customary law of each Dayak customary area in KMNP that has a purpose to harmonize the relation of the forest to people and people to people. They applied for fines for the violation, for example, people must precede a permit prior to harvest the timber or non-timber to the customary council, and will be suspended when the activity threat the forest or social situation. Therefore, customary law has strong acknowledged here that people have conscience of its integrity to their lives which depend on the forest, and it can refer to the sustainability of the biodiversity in KMNP. This situation supported by the statement from Primack (2001) that customary act imposes restrictions to prevent uncontrolled exploitation of natural resources. This attitude was the background of collaborative management in KMNP to conserve forest and wildlife habitat for the benefit to people by educational, research, tourism and recreational uses (MoF 2002a). Also is supported by the statement that biological conservation depends on the ecological behavior of human societies (Angermeier 2000). Seeing this, it is understandable that traditional ecological knowledge of medicinal plants of the Dayak linking to supporting one another with biodiversity conservation in KMNP. The linkage concept is proposed for the local government and the managers of the park, in Figure 4, in developing KMNP to collaborate the traditional knowledge and biodiversity conservation which will support for a sustainable income for the villagers in the future.

This linkage concept explains less significant adverse effects to the biodiversity conservation in KMNP in involving public participation, while the legal status of this area as national park supports acknowledging customary areas from threats possibly caused from exploiting policy or free riders.

In conclusion, the research found that young people still have good knowledge and actively use medicinal plants species to the present day is a good sign. Still, there is a threat to this traditional knowledge since more young people leave their villages for formal education that possibly preclude from gaining such knowledge, also no apprenticeship currently by local healer and elderly knowledgeable villagers. This situation could cause a loss of knowledge as it will disappear by the passes of the local healer or knowledgable elders if not shared. KMNP management will have to emphasize traditional ecological knowledge to a short or long-term management plan to enhance understanding between people and national park to achieve the objective in collaborative management. Stakeholders in KMNP are expected to support more in communicating the traditional ecological knowledge and biodiversity conservation in the community since an early age to nurture more respect from younger people as they will act as future leaders and will need good conservation vision. Therefore, local environmental education and apprenticeship must also be encouraged to minimize the gap between older and younger people, and to prevent the loss of this knowledge. Some precautionary approaches of sustainability are needed in future development after noticing those important medicinal plants species of trees 
have to function for timber as well. Further research is needed in continuous way the broader aspects of traditional ecological knowledge of medicinal plants species of Dayak Krayan people and biodiversity conservation in the area as this research focused only on two villages for time range of last three years, in order to create the database information of Dayak people's medicinal plants species in KMNP. Such a database of knowledge, species, and abundance are important for the management of biological diversity regarding the dynamics of the people and the development in Indonesia.

\section{ACKNOWLEDGEMENTS}

Acknowledgment to Russell E. Train fellowship and ITTO for financial support, Daniel Kapung, villagers of Wa' Yagung and Pa' Padi in Krayan, WWF-KM, and Ministry of Forestry of Indonesia for kind support and technical assistance. This article is based on the master thesis of the first author. Profound gratitude to Prof. Dr. rer. nat. habil. Dr. h.c. Peter A. Schmidt (the main supervisor at Technische Universität Dresden, Germany, retired in 2014) and Prof. Tomohiro Ichinose (current supervisor) for the encouragement to publish it. Some parts of this research support her current doctoral research at Keio University, Japan.

\section{REFERENCES}

Angermeier PL. 2000. The natural imperative for biological conservation. Conserv Biol 14: 373-381.

Balick MJ, Arvigo R, Shropshire G, Mendelsohn R. 1996 Ethnopharmacological studies and biological conservation in Belize (pp.326-333). In: Balick MJ, Elisabetsky E, Laird SA (eds). Medicinal Resources of the Tropical Forest, Biodiversity and Its Importance to Human Health. Columbia University Press, New York.

Balick MJ, Cox PA. 1997. Ethnobotanical research and traditional health care in developing countries (pp.12-23). In: Bodeker G, Bhat KKS, Burley J, Vantomme P (eds). Medicinal Plants for Forest Conservation and Health Care. Non-Wood Forest Products 11. FAO, Rome.

Berkes F. 1993. I. Traditional ecological knowledge in perspective. In: Inglis JT (eds) Traditional Ecological Knowledge: Concepts and Cases. International Development Research Center, Ottawa.

Bleher B, Uster D, Bergsdorf T. 2006. Assessment of threat status and management effectiveness in Kakamega forest, Kenya. Biodivers Conserv 15: 1159-1177. In: Hawksworth DL, Bull AT (eds). Topics in Biodiversity and Conservation Volume 2: Forest Diversity and Management.

Caniago I, Siebert SE. 1998. Medicinal plant ecology, knowledge and conservation in Kalimantan, Indonesia. Econ Bot 52 (3): 229-250.

Cook FEM. 1995. Economic Botany: Data Collection Standard. Prepared for the International Working Group on Taxonomic Databases for Plant Sciences (TDWG). Royal Botanic Gardens, Kew.

Cutler DF, Botha CEJ, Stevenson DW. 2008. Plant Anatomy: An Applied Approach. Blackwell Publishing, Carlton.

Fitter AH, Hay RKM. 2002. Environmental Physiology of Plants. 3rd ed. Academic Press, San Diego.

Foote LE, Jones Jr SB. 1989. Native Shrub and Woody Vines of the Southeast: Landscaping Uses and Identification. Timber Press, Portland.

Furze B, De Lacy T, Brickhead J. 1996. Culture, Conservation and Biodiversity. John Wiley \& Sons, Chichester.

Gerique A. 2006. An introduction to ethnoecology and ethnobotany: integrative assessment and planning methods for sustainable agroforestry in humid and semiarid regions. Advanced Scientific Training, Loja, Ecuador.

Ghimire K, Bastakoti RR. 2009. Ethnomedicinal knowledge and healthcare practices among the Tharus of Nawalparasi District in Central Nepal. For Ecol Manag 257: 2066-2072.

Harding DJ, Lefsky MA, Parker GG, Blair JB. 2001. Laser altimeter canopy height profiles: Methods and validation for closed-canopy, broadleaf forests. Remote Sens Environ 76 (3): 283-297.

Iiyama Y. 2003. Community Perspectives on Ecotourism Carrying Capacity: Four villages in Pujungan Sub-district, Kayan Mentarang National Park, East Kalimantan, Indonesia Case Study. [Thesis]. King's College-London University, London.

Johannes RE. 1989. Introduction (pp.5-9). In: Johannes RE (ed) Traditional Ecological Knowledge: A Collection of Essays. IUCN, Gland, UK.

Johnson M. 1992. Research on traditional environmental knowledge: its development and its role. In: Johnson M (ed) Lore: Capturing Traditional Environmental Knowledge. Dene Cultural Institute/International Development Research Center, Ottawa.

Kala CP. 2000. Status and conservation of rare and endangered medicinal plants in the Indian trans-Himalaya. Biol Conserv 93: 371-379. DOI: 10.1016/S0006-3207 (99)00128-7

Kathe W. 2006. Chapter 8 Revision of the 'guidelines on the conservation of medicinal plants BY WHO, IUCN, WWF and TRAFFIC: process and scope. In: Bogers RJ, Craker LE, Lange D. (eds) Medicinal and Aromatic Plants. Springer, Netherlands.

KSDG. 2009. Krayan Sub-district Government Monthly Report Book.

Langub J. 2005. An overview of wilayah adat Krayan Darat and Krayan Hilir. In: Topp L, Eghenter C (eds) Kayan Mentarang National Park in the Heart of Borneo. WWF-Indonesia, Jakarta.

Larcher W. 1995. Photosynthesis as a tool for indicating temperature stress events. In: Ecophysiology of photosynthesis. Springer, Berlin.

Leaman DJ, Arnason JT, Yusuf R, Sangat-Roemantyo H, Soedjito H, Angerhofer CK, Pezzuto JM. 1995. Malaria remedies of the Kenyah of the Apo Kayan, East Kalimantan, Indonesian Borneo: a quantitative assessment of local consensus as an indicator of biological efficacy. J Ethnopharmacol 49: 1-16. DOI: 10.1016/03788741 (95)01289-3

MacKinnon K, Hatta G, Halim H, Mangalik A. 1996. The Ecology of Kalimantan: Indonesian Borneo. Periplus Edition (HK) Ltd. Oxford University Press.

Martin GJ. 1998. Ethnobotani: Satu Manual Kaedah. (translated by Maryati Mohamed). Natural History Publications, Kota Kinabalu in cooperation with WWF International, Gland. [Malays]

Matius P, Tipot E, Kuda T. 2003. Chapter 28 Ethnobotany of the Lundayeh community in $\mathrm{Pa}$ Raye village, East Kalimantan. In: Mardiastuti A, Soehartono T (eds). Joint Biodiversity Expedition in Kayan Mentarang National Park Report. (official e-book report in the compact disc).

Mayers J, Bass S. 1998. The role of policy and institutions. In: Goldsmith FB (eds) Tropical Rain Forest: A Wider Perspective. Chapman and Hall, London

McNeely JA, MacKinnon JR. 1990. Protected areas, development, and land use in the tropics. Resour Manag Optimization 7 (1-4): 191-208. In: Furtado JI, Morgan WB, Pfafflin JR, Ruddle K (eds) Tropical Resources: Ecology and Development. Harwood Academic Publishers, Chur.

MoF. 2002a. Kayan Mentarang National Park Management Plan. Book I: Management Plan. Ministry of Forestry of Indonesia, Jakarta.

MoF. 2002b. Kayan Mentarang National Park Management Plan. Book II: Data, Projection, and Analysis. Ministry of Forestry of Indonesia, Jakarta.

MoF. 2007. Rencana pengelolaan jangka menengah (RPJM) Taman Nasional Kayan Mentarang. Ministry of Forestry of Indonesia, Jakarta.

Montagnini F, Jordan CF. 2005. Tropical Forest Ecology: The Basis for Conservation and Management. Springer, Berlin.

Morgan GA, Leech NL, Gloeckner GW, Barret KC. 2007. SPSS for Introductory Statistics: Use and Interpretation. 3rd ed. Lawrence Erlbaum Associates Inc. Publishers, New Jersey.

Mosbrugger V. 1990. The tree habit in land plants: A functional comparison of trunk constructions with a brief introduction into the biomechanics of trees. In: Bhattacharji S, Friedman GM, Neugebauer HJ, Seilacher A (eds). Lecture Notes in Earth Sciences 28. SpringerVerlag, Berlin. 
Mueller MS, Mechler E. 2005. Medicinal Plants in Tropical Countries. Thieme, Stuttgart.

Odum EP. 1969. The Strategy of Ecosystem Development. Sci New Ser 164 (3877): 262-270.

Posey DD. 1997. Wider use and application of indigenous knowledge, innovations and practices: information systems and ethical concerns (pp.69-103). In: Hawksworth DL, Kirk PM, Clarke SD (eds) Biodiversity Information: Needs and Options: Proceedings of the 1996 International Workshop on Biodiversity Information. CAB International, Oxon.

Primack RB. 2001. Extinction, Cause of. Encyclopedia of Biodiversity Volume 2. Academic Press, San Diego, CA.

Sangat HM, Zuhud EAM, Damayanti EK. 2000. Kamus Penyakit dan Tumbuhan Obat Indonesia. Yayasan Obor Indonesia, Jakarta. [Indonesian]

SCBD. 2006. Global Biodiversity Outlook 2. Secretariat of the Convention on Biological Diversity, Montreal.

Schippmann U, Leaman D, Cunningham AB. 2006. Chapter 6 A comparison of cultivation and wild collection of medicinal and aromatic plants under sustainability aspects. In: Bogers RJ, Craker LE, Lange D (eds). Medicinal and Aromatic Plants. Springer, Netherlands.

Schneeberger WF. 1945. The Kerayan-Kalabit highland of Central Northeast Borneo. Geogr Rev 35 (4): 544-562.

Schnitzer SA, Bongers F. 2002. The ecology of lianas and their role in forests. Trends Ecol Evol 17 (5): 223-230.

Sedjo RA. 2000. Biodiversity: forests, property rights and economic value. In: Van Kooten GC, Bulte EH, Sinclair ARE (eds). Conserving Nature's Diversity: Insights from Biology, Ethics and Economics. Ashgate Publishing Limited, Hampshire.

Sheldon JW, Balick MJ. 1995. Ethnobotany and the search for balance between use and conservation (pp.45-64). In: Swanson TM (ed). Intellectual Property Rights and Biodiversity Conservation, An Interdisciplinary Analysis of the Values of Medicinal Plants. Cambridge University Press, Cambridge.
Townsley P. 1996. 3. What is rapid rural appraisal? Rapid rural appraisal, participatory rural appraisal and aquaculture. FAO Fisheries Technical Paper-T358, FAO, Rome.

Turner IM. 2001. The Ecology of Trees in the Tropical Rain Forest. Cambridge University Press, Cambridge.

Velasco-Lezama R, Tapia-Aguilar R, Román-Ramos R, Vega-Avila E, Pérez-Gutiérrez MS. 2006. Effect of Plantago major on cell proliferation in vitro. J Ethnopharmacol 103 (1): 36-42.

WCMC (World Conservation Monitoring Center).1998. Alstonia scholaris. The IUCN Red List of Threatened Species 1998: e.T32295A9688408.DOI:10.2305/IUCN.UK.1998.RLTS.T32295A96 88408.en.

Wulffrat S. 2005. An ecological overview of Krayan Darat and Krayan Hilir. In: Topp L, Eghenter C (eds) Kayan Mentarang National Park in the Heart of Borneo. WWF-Indonesia, Jakarta.

WWF. 2007. Borneo montane rain forests. In: McGinley M (ed). Encyclopedia of Earth. Cleveland CJ (eds). Environmental Information Coalition, National Council for Science and the Environment, Washington, D.C.

Yuliarti N. 2008. Hidup Sehat dengan Terapi Herbal. Banyu Media, Yogyakarta. [Indonesian]

Zuhud EAM. 2007. Tri-Stimulus AMAR (Alamiah, MAnfaat, Religius) pro-konservasi "Three-Stimulus NUR (Natural-Usefulness-Religion) pro-conservation" (suatu konsep teori sikap dan perilaku prokonservasi yang digali dari budaya masyarakat asli Indonesia). Buku prosiding Lokakarya Pendidikan Konservasi IPB-RARE, Bogor. [Indonesian]

Zuhud EAM, Sofyan K, Prasetyo LB, Kartodihardjo H. 2007. Sikap masyarakat dan konservasi, suatu analisis Kedawung (Parkia timoriana (DC) Merr.) sebagai stimulus tumbuhan obat bagi masyarakat: kasus di Taman Nasional Meru Betiri. Media Konservasi, Jurnal Ilmiah Bidang Konservasi Sumberdaya Alam Hayati dan Lingkungan 12 (2): 20pp. [Indonesian] 